\title{
Aplicação de um Comitê de Redes Neurais Artificiais para a Solução de Problemas Inversos em Transferência Radiativa ${ }^{1}$
}

R,C. OLIVEIRA ${ }^{2}$, N.I.A. ACEVEDO ${ }^{3}$, A.J. SILVA NETO ${ }^{4}$ Instituto PolitécnicoIPRJ, Universidade do Estado do Rio de Janeiro, Cx.P. 97282, 28601-970 Nova Friburgo, RJ, Brasil

L. BIONDI NETO ${ }^{5}$, Universidade do Estado do Rio de Janeiro-UERJ, Rua São Francisco Xavier, 524, 200550-013, Maracanã, Rio de Janeiro, RJ, Brasil.

Resumo. Este trabalho fundamenta-se no conceito de Máquina de Comitê de Redes Neurais Artificiais e tem por objetivo resolver o problema inverso de transferência radiativa em um meio unidimensional, homogêneo, absorvedor e espalhador isotrópico. A Máquina de Comitê de Redes Neurais Artificiais agrega e combina o conhecimento adquirido de um certo número de especialistas aqui representados, individualmente, por cada uma das Redes Neurais Artificiais (RNAs) que compõem o Comitê. O objetivo é atingir um resultado final hipoteticamente melhor que o obtido por qualquer rede neural especialista separadamente. $\mathrm{O}$ uso desta técnica pode reduzir o desperdício computacional que ocorre ao se treinar exaustivamente várias RNAs, separadamente, selecionando-se apenas a rede que apresente a melhor generalização e descartando-se as demais. Neste trabalho são obtidas, usando-se a técnica de Máquina de Comitê de Redes Neurais Artificiais, estimativas de parâmetros de transferência radiativa: espessura óptica, albedo de espalhamento simples e reflectividades difusas do meio participante sob análise. Finalmente, os resultados obtidos são comparados com os encontrados usando-se redes Perceptrons de Múltiplas Camadas (PMCs) individualmente, denominadas neste trabalho como redes especialistas e mostrando que a técnica empregada traz significativas melhorias de desempenho com custo computacional relativamente baixo.

Palavras-chave. Transferência radiativa, Problemas inversos, Máquina de comitê, Perceptron de Múltiplas Camadas, Redes neurais artificiais.

\footnotetext{
${ }^{1}$ Uma versão preliminar deste trabalho foi apresentada no XI Encontro de Modelagem Computacional, Volta Redonda, RJ, 2008 [7].

${ }^{2}$ rcoliveira@iprj.uerj.br

${ }^{3}$ nacevedo@iprj.uerj.br

4ajsneto@iprj.uerj.br

${ }^{5}$ luizbiondi@terra.com.br
} 


\section{Introdução}

A análise do fenômeno de transferência radiativa em meios participantes tem atraído a atenção de muitos pesquisadores devido a sua relevante aplicação em várias áreas tais como avaliação de risco de incêndios, modelos climáticos regionais e globais [1-4, 10-13].

Com o objetivo de reduzir o tempo computacional para a solução dos problemas inversos de transferência radiativa para a estimativa de propriedades radiativas em meios participantes homogêneos e heterogêneos, têm sido usados redes neurais artificiais [1, 3] e métodos híbridos [10, 11, 13].

No presente trabalho a formulação e a solução do problema inverso de transferência radiativa para a estimativa da espessura óptica $\tau_{0}$, do albedo de espalhamento simples $\omega$, e das reflectividades difusas $\rho_{1}$ e $\rho_{2}$, num meio unidimensional, participante e homogêneo, aplicando uma Máquina de Comitê de Redes Neurais Artificiais é apresentada $[6,7]$. Os padrões usados no treinamento da máquina de comitê foram gerados com a solução da versão linear da equação de Boltzmann, que é usada para modelar o problema direto de transferência radiativa.

A máquina de comitê de redes neurais investigada (Fig. 1) é formada por dez Redes Neurais Artificiais (RNAs) especialistas do tipo PMC (Perceptron de Múltiplas Camadas) com arquitetura e parâmetros de treinamento distintos, e compartilhando as mesmas entradas. As saídas dessas dez redes PMC são adequadamente preparadas e servem de entrada para o módulo combinador, representado por uma única rede PMC, fornecendo na saída o resultado final da rede de comitê que representa as estimativas para as quatro propriedades do meio participante. As entradas das redes especialistas recebem dados experimentais, $Y_{i}, i=1, \ldots, N$, onde $N$ representa o número total de dados experimentais. Na camada de saída de cada rede neural especialista (REDE ESPECIALISTA $1, \ldots$, REDE ESPECIALISTA $_{10}$ ) são colhidos os resultados relativos às propriedades do meio que serão combinados no estágio final da máquina de comitê. Na saída da Máquina de Comitê, o COMBINADOR com quatro neurônios, um para cada uma das incógnitas, i.e., a espessura óptica, o albedo de espalhamento simples e as duas reflectividades, apresenta o resultado final do comitê de RNAs.

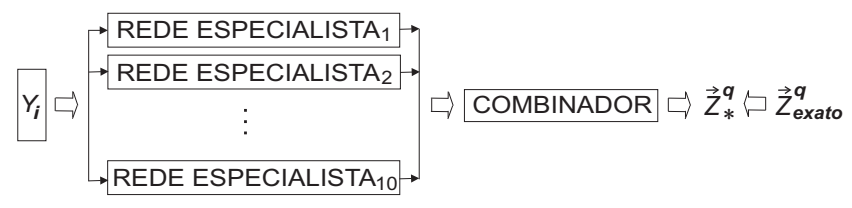

Figura 1: Estrutura da Máquina de Comitê de Redes Neurais Artificiais.

Como dados experimentais reais não estavam disponíveis, simulamos dados sintéticos adicionando um ruído pseudo-aleatório, às intensidades da radiação emergente, com dependência no ângulo polar, calculadas a partir da solução do problema direto de transferência radiativa usando os valores exatos dos parâmetros que são considerados como incógnitas no problema inverso [12].

Foram gerados, usando-se o modelo computacional para o problema direto de 
transferência radiativa, dez mil padrões de treinamento formados por dois conjuntos de informações: intensidade da radiação que deixa o meio e parâmetros de transferência radiativa do meio. Esses sinais, que são os dados de entrada do comitê, excitam as dez redes neurais especialistas, independentemente umas das outras, que em conseqüência produzem as saídas que são transmitidas como entradas à rede neural combinadora das saídas das redes neurais especialistas da Máquina de Comitê, que, por conseguinte, agrega e combina esses resultados, mapeando-os sobre os mesmos alvos usados no treinamento das redes neurais especialistas. No caso pesquisado a rede neural de saída da Máquina de Comitê é composta por quatro sub-redes, uma para cada um dos parâmetros radiativos estimados. Nos testes de execução, visando verificar a generalização da Máquina de Comitê, foram utilizados dados que não participaram do treinamento.

Os resultados obtidos com a máquina de comitê são então comparados com os resultados obtidos pelas redes neurais especialistas individualmente, demonstrando o desempenho superior na solução do problema inverso de transferência radiativa.

\section{Formulação Matemática e Solução do Problema Direto}

Considere um meio unidimensional, homogêneo e participante, i.e., absorvedor e espalhador isotrópico, como mostrado na Fig. 2.

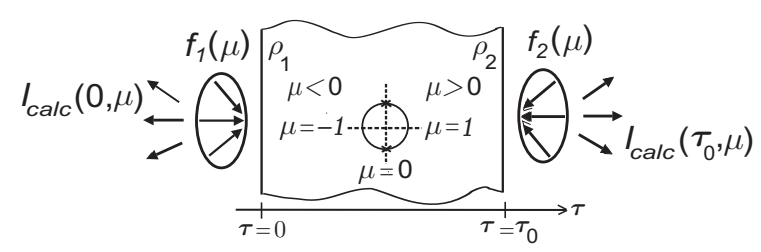

Figura 2: Meio participante unidimensional homogêneo.

A formulação matemática do problema de transferência radiativa é feita com a versão linear da equação de Boltzmann, também conhecida como equação de transporte, que pode ser escrita na seguinte forma adimensional, assumindo simetria azimutal [8],

$$
\mu \frac{\partial I(\tau, \mu)}{\partial \tau}+I(\tau, \mu)=\frac{\omega}{2} \int_{-1}^{1} I\left(\tau, \mu^{\prime}\right) d \mu^{\prime} \quad \text { em } \quad 0<\tau<\tau_{0},-1 \leq \mu \leq 1,
$$

onde $I$ é a intensidade da radiação, $\tau$ é a variável óptica adimensional, $\mu$ é o co-seno do ângulo polar $\theta$ (ângulo entre o eixo - $\tau$ positivo e a direção do feixe de radiação), usado para descrever a direção de propagação da radiação, e $\omega$ é o albedo de espalhamento simples, que corresponde à razão entre o coeficiente de espalhamento, $\sigma_{s}$, e o coeficiente de extinção, $\beta$. 
Para a formulação e solução dos problemas diretos de transferência radiativa acrescentamos à Eq. (2.1) as condições de contorno

e

$$
\begin{aligned}
I(0, \mu) & =f_{1}(\mu)+2 \rho_{1} \int_{0}^{1} I\left(0,-\mu^{\prime}\right) \mu^{\prime} d \mu^{\prime}, \quad \mu>0 \\
I\left(\tau_{0}, \mu\right) & =f_{2}(\mu)+2 \rho_{2} \int_{0}^{1} I\left(\tau_{0}, \mu^{\prime}\right) \mu^{\prime} d \mu^{\prime}, \quad \mu<0 .
\end{aligned}
$$

Os valores $f_{1}(\mu)$ e $f_{2}(\mu)$ são as intensidades da radiação externa incidente, respectivamente, em $\tau=0$ e $\tau=\tau_{0}$, e $\rho_{1}$ e $\rho_{2}$ são as reflectividades difusas nas superfícies em $\tau=0$ e $\tau=\tau_{0}$, na parte interna do meio. A espessura óptica $\tau_{0}$ e o albedo de espalhamento simples $\omega$ são dados por

$$
\tau_{0}=\beta L \quad \text { e } \quad \omega=\sigma_{s} / \beta
$$

com o coeficiente de extinção igual a $\beta=\kappa_{a}+\sigma_{s}$, onde $\kappa_{a}$ e $\sigma_{s}$ representam respectivamente os coeficientes de absorção e de espalhamento do material, e $L$ é a espessura física do meio.

Para a solução do problema direto utilizamos no presente trabalho uma combi-

nação do método das ordenadas discretas [5] com o método das diferenças finitas [13].

\section{Formulação Matemática e Solução do Problema Inverso com Máquina de Comitê de Redes Neu- rais}

Estamos interessados na obtenção das estimativas para o vetor de incógnitas

$$
\vec{Z}=\left\{\tau_{0}, \omega, \rho_{1}, \rho_{2}\right\}
$$

usando os dados medidos sobre a intensidade da radiação emergida em $\tau=0$ e $\tau=\tau_{0}, Y_{i} \operatorname{com} i=1,2, \ldots, N$, sendo $N$ o número total de dados experimentais. Aqui será usado $N=20$.

Como dados experimentais reais não estão disponíveis, é construído um conjunto de dados experimentais sintéticos com

$$
Y_{i}=I_{\text {calc }_{i}}\left(\vec{Z}_{\text {exato }}\right)+\sigma r_{i}, \quad i=1,2, \ldots, N,
$$

onde $I_{\text {calc }_{i}}$ representa os valores calculados da intensidade da radiação usando os valores exatos das propriedades radiativas, que numa aplicação real não estão disponíveis, as quais queremos determinar com a solução do problema inverso, $\sigma$ simula o desvio padrão dos erros dos dados experimentais, e $r_{i}$ é um número pseudo-aleatório gerado no intervalo $[-1,1]$.

Com o objetivo de solucionar o problema inverso de transferência radiativa é usada aqui uma Máquina de Comitê de Redes Neurais, conforme representado esquematicamente na Fig. 1, composta por dez PMCs (Perceptron de Múltiplas Camadas), redes neurais especialistas, e um PMC com uma camada oculta e uma 
camada de saída com quatro neurônios, o combinador, que fornece a saída da máquina de comitê. Na Fig. 3 está representado um PMC para a solução do problema inverso em transferência radiativa, com uma camada de entrada, uma camada de saída com quatro neurônios $\left(N_{u n}=4\right)$, um para cada parâmetro radiativo a ser determinado, e duas camadas ocultas (neste trabalho também foram usados PMCs com apenas uma camada oculta).

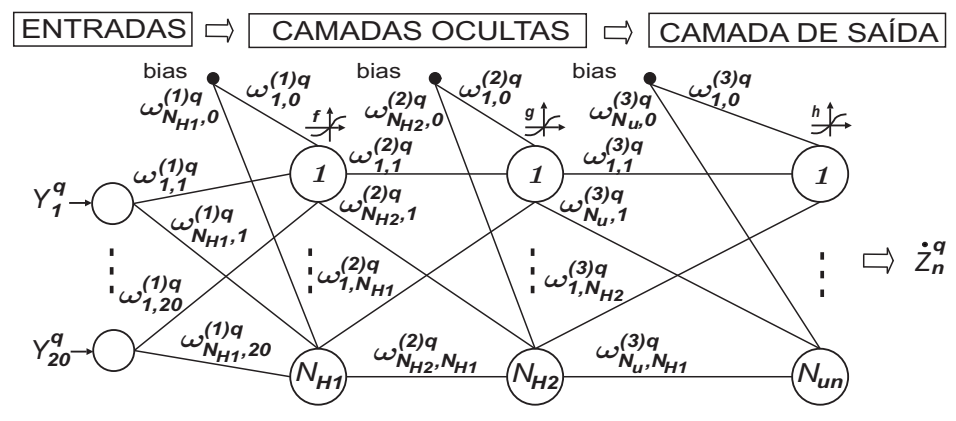

Figura 3: Perceptron de Múltiplas Camadas para o problema inverso, com duas camadas escondidas.

Na Fig. 4 está representado um PMC simplificado, com uma camada oculta com $N_{h}$ neurônios, e uma camada de saída com um neurônio apenas, especializado em calcular um único parâmetro de radiação. Este PMC decorre do desmembramento do PMC de saída da máquina de comitê em quatro PMCs simplificados (sub-redes) utilizando a topologia descrita anteriormente. Com esta topologia, constrói-se a máquina de comitê de redes neurais para solucionar o problema inverso de transferência radiativa, determinando o vetor dos parâmetros $\vec{Z}$, Eq. (3.1), a partir do conhecimento das intensidades de radiação $Y_{i}, i=1,2, \ldots, N$, obtidas de acordo com a Eq. (3.2).

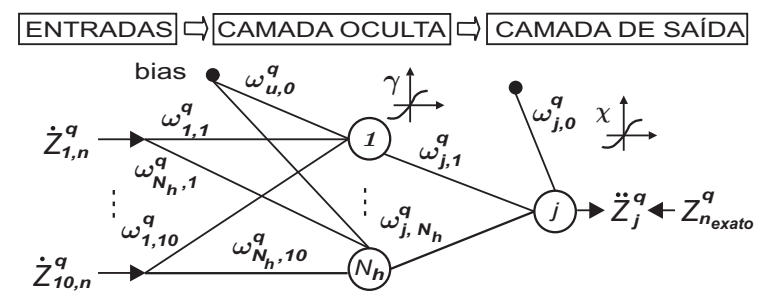

Figura 4: Modelo simplificado do PMC de saída com uma camada oculta da máquina de comitê.

as redes neurais especialistas representadas na Fig. 3 cada neurônio $m$, com $m=1,2, \ldots, N_{H 1}$, na primeira camada oculta realiza uma combinação linear dos valores de entrada $Y_{l}, \operatorname{com} l=1,2, \ldots, N$, fornecidas pela camada de entrada

$$
a_{m}^{q}=\sum_{l=1}^{N} \omega_{m, l}^{(1) q} Y_{l}+w_{m, 0}^{(1) q}, \quad m=1,2, \ldots, N_{H 1},
$$


onde $\omega_{m, l}^{(1) q}, m=1,2, \ldots, N_{H 1}, l=1,2, \ldots, N$, são os pesos sinápticos que conectam os nós das entradas com os neurônios da primeira camada oculta, $N$ é o número de nós na camada de entrada, sendo aqui considerado $N=20, N_{H 1}$ é o número de neurônios da primeira camada oculta, e o índice $q$ representa a ordem do padrão de treinamento que está entrando no comitê, $q=0, \ldots, Q-1$, onde aqui $Q=10.000$.

A soma ponderada $a_{m}^{q}$ dada pela Eq. (3.3) é vista como uma excitação para o neurônio $m$ da primeira camada oculta, que fornece a resposta

$$
p_{m}^{q}=f\left(a_{m}^{q}\right),
$$

onde $f(\cdot)$ é uma função de ativação. Várias escolhas para a função $f(\cdot)$ são possíveis [6].

Cada neurônio $k$, com $k=1,2, \ldots, N_{H 2}$, na segunda camada oculta realiza uma combinação linear dos valores das respostas $p_{m}^{q}, m=1,2, \ldots, N_{H 1}$, fornecidas pela primeira camada oculta

$$
b_{k}^{q}=\sum_{m=1}^{N_{H 1}} \omega_{k, m}^{(2) q} p_{m}^{q}+w_{k, 0}^{(2) q}, \quad k=1,2, \ldots, N_{H 2},
$$

onde $\omega_{k, m}^{(2) q}, k=1,2, \ldots, N_{H 2}, m=1,2, \ldots, N_{H 1}$, são os pesos sinápticos que conectam os neurônios da primeira camada oculta com os neurônios da segunda camada oculta.

A soma ponderada $b_{k}^{q}$ dada pela Eq. (3.5) é vista como uma excitação para o neurônio $k$ da segunda camada oculta, que fornece a resposta

$$
\nu_{k}^{q}=g\left(b_{k}^{q}\right), \quad k=1,2, \ldots, N_{H 2},
$$

onde $g(\cdot)$ é uma função de ativação. Várias escolhas para a função $g(\cdot)$ são possíveis [6].

Cada neurônio $n$ da camada de saída, $\operatorname{com} n=1,2, \ldots, N_{u n}$, realiza uma combinação linear das respostas $\nu_{k}^{q}, k=1,2, \ldots, N_{H 2}$, dos neurônios da segunda camada oculta,

$$
s_{n}^{q}=\sum_{k=1}^{N_{H 2}} \omega_{n, k}^{(3) q} \nu_{k}^{q}+w_{n, 0}^{(3) q}, \quad n=1,2, \ldots, N_{u n},
$$

onde $\omega_{n, k}^{(3) q}, n=1,2, \ldots, N_{u n}, k=1,2, \ldots, N_{H 2}$, são os pesos sinápticos entre os neurônios da segunda camada oculta e os neurônios da camada de saída, e $N_{u n}$ é o número de neurônios na camada de saída, que coincide com o número de incógnitas do problema inverso. Aqui temos $N_{u n}=4$, conforme a Eq. (3.1).

A soma ponderada $s_{n}^{q}$ dada pela Eq. (3.7) é vista como uma excitação para o neurônio $n$ da camada de saída, que produz uma resposta

$$
t_{n}^{q}=h\left(s_{n}^{q}\right), \quad n=1,2, \ldots, N_{u n},
$$

onde $h(\cdot)$, é uma função de ativação. Várias escolhas para a função $h(\cdot)$, são possíveis [6]. 
Combinando as Eqs. (3.3) a (3.8), para duas camadas ocultas, obtemos

$$
\begin{array}{r}
t_{n}^{q}=h\left(\sum_{k=1}^{N_{H 2}} \omega_{n, k}^{(3) q} g\left(\sum_{m=1}^{N_{H 1}} \omega_{k, m}^{(2) q} f\left(\sum_{l=1}^{N} \omega_{m, l}^{(1) q} Y_{l}+\omega_{m, 0}^{(1) q}\right)+\omega_{k, 0}^{(2) q}\right)+\omega_{n, 0}^{(3) q}\right) \\
n=1,2, \ldots, N_{u n} .
\end{array}
$$

Considerando disponíveis os dados experimentais $Y_{l}, l=1,2, \ldots, N$, observamos na Eq. (3.9) que $t_{n}^{q}, n=1,2, \ldots, N_{u n}$, são as estimativas, $\dot{Z}_{n}^{q}$, com $n=1,2, \ldots, N_{u n}$, obtidas pela rede neural especialista. Porém, antes que possamos usar a Eq. (3.9) devemos determinar os pesos sinápticos $\omega^{(1) q}, \omega^{(2) q}$ e $\omega^{(3) q}$.

A determinação dos pesos $\omega^{(1) q}, \omega^{(2) q}$ e $\omega^{(3) q}$ é feita pela apresentação de um conjunto de padrões (entradas conhecidas $\vec{Y}_{\text {exato }}$ e saídas desejadas $\vec{Z}_{\text {exato }}$ ) e calculamse os pesos que produzam o melhor ajuste entre os valores de $\vec{t}$ calculado e o vetor das saídas desejadas $\vec{Z}_{\text {exato }}$. Os padrões usados no treinamento supervisionado das RNAs foram gerados calculando-se os valores $\vec{Y}_{\text {exato }}$ a partir do vetor exato dos parâmetros $\vec{Z}_{\text {exato }}$ com o método das ordenadas discretas e a solução por diferenças finitas mencionados na seção anterior $[5,10]$.

Para a determinação de $\omega^{(1) q}, \omega^{(2) q}$ e $\omega^{(3) q}$ é usado o algoritmo de retropropagação. Inicia-se com uma estimativa inicial para os pesos $\omega^{(1) q}, \omega^{(2) q}$ e $\omega^{(3) q}$, com $q=0$, e o conjunto de entradas $\vec{Y}$ é passada para frente (feed forward) através da rede produzindo estimativas de saídas $\vec{t}^{q=0}$ que serão comparadas com as saídas desejadas $\vec{Z}_{\text {exato }}$ produzindo os erros,

$$
e_{n}^{q}=Z_{\text {exaton }_{n}}-t_{n}^{q}, \quad n=1,2, \ldots, N_{u n} .
$$

Os pesos são então ajustados usando a informação fornecida pelo erro da saída

$$
\begin{array}{llll}
\omega_{n, k}^{(3) q+1}=\omega_{n, k}^{(3) q}+\eta \delta_{n}^{(3) q} \nu_{k}^{q}, & \text { onde } & \delta_{n}^{(3) q}=e_{n}^{q} h^{\prime}\left(s_{n}^{q}\right) \\
\omega_{k, m}^{(2) q+1}=\omega_{k, m}^{(2) q}+\eta \delta_{k}^{(2) q} p_{m}^{q}, & \text { onde } & \delta_{k}^{(2) q}=g^{\prime}\left(b_{k}^{q}\right) \sum_{n=1}^{N_{u n}} \delta_{n}^{(3) q} \omega_{n, k}^{(3) q} \\
\omega_{m, l}^{(1) q+1}=\omega_{m, l}^{(1) q}+\eta \delta_{m}^{(1) q} Y_{l}^{q}, & \text { onde } & \delta_{m}^{(1) q}=f^{\prime}\left(a_{m}^{q}\right) \sum_{k=1}^{N_{H 2}} \delta_{k}^{(2) q} \omega_{k, m}^{(2) q}
\end{array}
$$

e $\eta$ é a taxa de aprendizado, que pode assumir diferentes valores. Sendo que cada PMC utiliza um único valor de taxa de aprendizado no seu treinamento, e $h^{\prime}, g^{\prime}$ e $f^{\prime}$ representam as derivadas das funções de ativação.

Na camada oculta da PMC simplificada, componente da RNA de saída da máquina de comitê representada na Fig. 4 , o neurônio $u$, com $u=1,2 \ldots, N_{h}$, realiza parte do processamento dos valores dos resultados $\dot{Z}_{i, n}^{q}, n=1,2, \ldots, N_{u n}$, fornecidos pelas redes especialistas $\mathrm{PMC}_{i}, i=1,2, \ldots, M$, aqui $M=10$, através da Eq. (3.9), para cada propriedade radiativa individualmente

$$
z_{u}^{q}=\sum_{i=1}^{M} \omega_{u, i}^{q} \dot{Z}_{i, n}^{q}+\omega_{u, 0}^{q}, \quad u=1,2, \ldots, N_{h}, \quad i=1,2, \ldots, M,
$$


onde $\omega_{u, i}^{q}, u=1,2, \ldots, N_{h}, i=1,2, \ldots, M, q=0,1,2, \ldots, Q-1$, (aqui $Q=10.000$ : número de padrões de treinamento que correspondem a uma época) são os pesos sinápticos entre o neurônio $u$ da camada oculta da PMC de saída simplificada da máquina de comitê e os nós de suas entradas, que são as saídas dos especialistas, para cada parâmetro $\dot{Z}_{i, n}^{q}, n=1,2, \ldots, N_{u n}, i=1,2, \ldots, M$, individualmente.

A soma ponderada $z_{u}^{q}$ dada pela Eq. (3.11) é vista como uma excitação para o neurônio $u$, que fornece a resposta

$$
\hat{z}_{u}^{q}=\gamma\left(z_{u}^{q}\right), \quad u=1,2, \ldots \ldots, N_{h},
$$

onde $\gamma(\cdot)$ é uma função de ativação. Várias escolhas para a função $\gamma($.$) são possíveis$ [6].

O neurônio $j$, da camada de saída, realiza uma combinação linear das respostas $\hat{z}_{u}^{q}, u=1,2, \ldots, N_{h}$, dos neurônios da camada oculta

$$
Z_{j}^{q}=\sum_{u=1}^{N_{h}} \omega_{j, u}^{q} \hat{z}_{u}^{q}+\omega_{j, 0}^{q}, \quad \text { com } \quad j=1,
$$

onde $\omega_{j, u}^{q}, j=1, u=1,2, \ldots, N_{h}$, são os pesos sinápticos entre os neurônios da camada oculta e o neurônio da camada de saída.

A soma ponderada $Z_{j}^{q}$ dada pela Eq. (3.13) é vista como uma excitação para o neurônio $j$ da camada de saída da PMC simplificada, que produz uma resposta

$$
\ddot{Z}_{j}^{q}=\chi\left(Z_{j}^{q}\right), \quad \text { com } \quad j=1,
$$

onde $\chi(\cdot)$ é uma função de ativação. Várias escolhas para a função $\chi(\cdot)$ são possíveis [6].

Para a determinação de $\omega_{u, i}^{q}$ e $\omega_{j, u}^{q}$ o procedimento é o mesmo utilizado para a obtenção dos pesos $w^{(1) q}, w^{(2) q}$ e $w^{(3) q}$ das RNAs especialistas, sendo que aqui as entradas são as saídas dos especialistas $\dot{Z}_{i, n}^{q}, i=1,2 \ldots, M$, com $M=10$, para cada propriedade radiativa representada pelo índice $n$, com $n=1,2, \ldots, N_{u n}$. Os erros são

$$
e_{j}^{q}=Z_{\text {exaton }}^{q}-\ddot{Z}_{j}^{q}, \quad \text { com } \quad j=1 .
$$

Os pesos são então ajustados usando a informação fornecida pelo erro da saída [6],

$$
\begin{aligned}
\omega_{j, u}^{q+1}=\omega_{j, u}^{q}+\dot{\eta} \delta_{j}^{q} \hat{z}_{u}^{q} & \text { onde } & \delta_{j}^{q}=e_{j}^{q} \chi^{\prime}\left(Z_{j}^{q}\right), \\
\omega_{u, i}^{q+1}=\omega_{u, i}^{q}+\dot{\eta} \delta_{u}^{q} \dot{Z}_{i, n}^{q} & \text { onde } & \delta_{u}^{q}=\gamma^{\prime}\left(z_{u}^{q}\right) \sum_{j=1}^{1} \delta_{j}^{q} \omega_{j, u}^{q},
\end{aligned}
$$

$\dot{\eta}$ é a taxa de aprendizado da PMC simplificada de saída do comitê, e $\chi^{\prime}$ e $\gamma^{\prime}$ representam as derivadas das funções de ativação.

\section{Resultados e Discussão}

As redes neurais especialistas estão divididas em dois grupos: um com uma camada oculta $\left(N_{H L}=1\right)$ e outro com duas camadas ocultas $\left(N_{H L}=2\right)$. A ferramenta 
computacional usada para desenvolvimento das Redes Neurais Artificiais foi a versão R2008a do programa MATLAB. As funções de ativação utilizadas são a sigmóide nas camadas ocultas e a linear na camada de saída nas PMCs com duas camadas ocultas. Nas PMCs com uma camada oculta é usada apenas a sigmóide, tanto na camada oculta quanto na de saída. A tolerância para o erro médio quadrático (EMQ) exigido é $10^{-5}$, e o número máximo de épocas, $N E=3000$. Foram usados valores de taxas de aprendizado $\eta$ entre 0,5 e 0,9 , exceto nos casos de taxa adaptativa, e a taxa de aprendizagem no combinador $\dot{\eta}=0,9$; as funções de treinamento: gradiente com momento, gradiente com momento e taxa de aprendizado adaptativa, e o algoritmo baseado no método de otimização de Levenberg-Marquardt, todas fundamentadas no algoritmo de retro-propagação de erro implementado neste trabalho.

Na Tabela 1 são mostradas as especificações e os resultados das redes neurais especialistas individualmente, para um vetor alvo $\vec{Z}_{\text {exato }}=\left\{\tau_{0} ; \omega ; \rho_{1} ; \rho_{2}\right\}^{T}=$ $\{1,0 ; 0,9 ; 0,1 ; 0,9\}^{T}$. São mostrados também o número de camadas ocultas, $N_{H L}$, e de neurônios, $N_{H}$, de cada uma delas, além dos erros relativos percentuais resultantes de cada um dos parâmetros radiativos: $E_{\tau_{0}}, E_{\omega}, E_{\rho_{1}}$ e $E_{\rho_{2}}$, obtidos com

$$
E_{n}=\left|\frac{Z_{\text {exato }_{n}}-Z_{\text {estimadon }_{n}}}{Z_{\text {exaton }_{n}}}\right| \times 100 \%, \quad n=1,2, \ldots, N_{\text {un }} .
$$

Verifica-se que os resultados obtidos para $\tau_{0}, \omega$ e $\rho_{2}$ são razoáveis, pois os erros relativos médios para cada um são respectivamente $6,1 \%, 3,59 \%$ e $5,0 \%$, ao passo que para $\rho_{1}$ os resultados, com o seu erro médio relativo em $99,27 \%$ não são de boa qualidade. Intencionalmente foi escolhido um caso teste difícil, com $f_{1}(\mu)=1.0$ e $f_{2}(\mu)=0$, nas Eqs. (2.2), o que leva a uma baixa sensibilidade ao parâmetro $\rho_{1}$.

Na Tabela 2 tem-se os resultados da Máquina de Comitê de Redes Neurais Artificiais, para o vetor alvo $\vec{Z}_{\text {exato }}=\left\{\tau_{0} ; \omega ; \rho_{1} ; \rho_{2}\right\}^{T}=\{1,0 ; 0,9 ; 0,1 ; 0,9\}^{T}$. Verifica-se que os resultados são bem melhores do que aqueles obtidos com as especialistas individualmente, embora para $\rho_{1}$ ainda se tenha uma estimativa de baixa qualidade, o que é inerente à situação física do problema tratado. Para os parâmetros $\tau_{0}, \omega$ e $\rho_{2}$ os erros relativos são respectivamente $0,51 \%, 0,07 \%$ e $0,31 \%$, e para o parâmetro $\rho_{1}$ o erro relativo é de $19,0 \%$.

O combinador das saídas das redes neurais especialistas da Máquina de Comitê possui 16 neurônios na camada escondida e quatro na de saída. Sendo que este PMC de saída está desmembrado em quatro PMCs, cada um com $N_{H}=4$ e um neurônio na camada de saída. Cada um destes PMCs é, portanto, especializado no cálculo de um único parâmetro radiativo.

\section{Conclusão}

Conclui-se que os resultados obtidos são apenas razoáveis para os PMCs especialistas e bons para a máquina de comitê, atingindo o objetivo pretendido que é a melhoria dos resultados obtidos com as redes neurais especialistas. Cabe aqui ressaltar, que é considerado um caso teste intencionalmente difícil, com baixa sensibilidade a uma das incógnitas, com $f_{1}(\mu)=1,0$ e $f_{2}(\mu)=0,0$. Neste caso o efeito da reflectividade difusa $\rho_{1}$ mantém-se nula na primeira marcha para frente, só contribuindo para o 
Tabela 1: Resultados das execuções das redes neurais especialistas para um vetor alvo $\vec{Z}_{\text {exato }}=\left\{\tau_{0} ; \omega ; \rho_{1} ; \rho_{2}\right\}^{T}=\{1,0 ; 0,9 ; 0,1 ; 0,9\}^{T}$ e nível de ruído de $8 \%(\sigma=0,002$ na Eq. (3.2)).

\begin{tabular}{|r|r|r|c|c|c|c|r|c|r|r|}
\hline RNA & $N_{H L}$ & $N_{H}$ & $\tau_{0}$ & $\omega$ & $\rho_{1}$ & $\rho_{2}$ & $\begin{array}{c}E_{\tau_{0}} \\
(\%)\end{array}$ & $\begin{array}{c}E_{\omega} \\
(\%)\end{array}$ & $\begin{array}{c}E_{\rho_{1}} \\
(\%)\end{array}$ & $\begin{array}{c}E_{\rho_{2}} \\
(\%)\end{array}$ \\
\hline \hline 1 & 1 & 8 & 0,9144 & 0,8536 & 0,2231 & 0,9397 & 8,6 & 5,2 & 123,1 & 4,4 \\
\hline 2 & 1 & 8 & 0,9445 & 0,8324 & 0,1980 & 0,8803 & 5,6 & 7,5 & 98,0 & 2,2 \\
\hline 3 & 1 & 10 & 1,0000 & 0,9350 & 0,1943 & 0,9076 & 0,0 & 3,9 & 94,3 & 0,8 \\
\hline 4 & 1 & 12 & 0,9449 & 0,9497 & 0,1073 & 0,9411 & 5,5 & 5,5 & 7,3 & 4,6 \\
\hline 5 & 1 & 12 & 1,0000 & 0,9077 & 0,2481 & 0,8962 & 0,0 & 0,9 & 148,1 & 0,4 \\
\hline 6 & 1 & 12 & 0,9147 & 0,9274 & 0,2401 & 0,9196 & 8,5 & 3,0 & 140,1 & 2,2 \\
\hline 7 & 2 & 4 & 0,8312 & 0,9478 & 0,2145 & 0,6138 & 16,9 & 5,3 & 114,5 & 31,8 \\
\hline 8 & 2 & 6 & 0,9982 & 0,9131 & 0,2412 & 0,9107 & 0,2 & 1,5 & 141,2 & 1,2 \\
\hline 9 & 2 & 8 & 1,1351 & 0,9000 & 0,1647 & 0,8882 & 13,5 & 0,0 & 64,7 & 1,3 \\
\hline 10 & 2 & 10 & 1,0226 & 0,9286 & 0,1614 & 0,8904 & 2,3 & 3,2 & 61,4 & 1,1 \\
\hline \hline
\end{tabular}

Tabela 2: Resultado da execução da máquina de comitê para um vetor alvo $\vec{Z}_{\text {exato }}=\left\{\tau_{0} ; \omega ; \rho_{1} ; \rho_{2}\right\}^{T}=\{1,0 ; 0,9 ; 0,1 ; 0,9\}^{T}$ e nível de ruído de $8 \%(\sigma=0,002$ na Eq. (3.2)).

\begin{tabular}{|c|c|c|}
\hline PARÂMETRO & RESULTADO & ERRO $(\%)$ \\
\hline \hline$\tau_{0}$ & 0,9949 & 0,51 \\
\hline$\omega$ & 0,9006 & 0,07 \\
\hline$\rho_{1}$ & 0,1190 & 19,0 \\
\hline$\rho_{2}$ & 0,8972 & 0,31 \\
\hline \hline
\end{tabular}

cálculo de $I_{\text {calc }}$ a partir da segunda marcha para frente. Considerando-se $f_{1}(\mu)=$ 1,0 e $f_{2}(\mu)=1,0$, o que representa um caso teste mais fácil, as estimativas dos valores das propriedades radiativas foram mais próximas dos valores exatos.

Como um dos possíveis desdobramentos do trabalho aqui apresentado as topologias da Máquina de Comitê e de seus componentes podem ser aprimoradas, como por exemplo, o não desmembramento do PMC de saída da máquina de comitê em quatro PMCs simplificados, incrementando, assim, a correlação entre os parâmetros radiativos e potencialmente influindo para obter melhores resultados. A utilização de outros algoritmos, dentre eles o método resiliente que faz parte de uma classe de estratégias rápidas de adaptação local para o treinamento, cujo algoritmo foi desenvolvido por Riedmiller e Braun [9], também deve ser investigada. Este é um método que independe da magnitude do gradiente do erro sobre o peso, no qual a atualização dos pesos depende, simplesmente, do sinal dos termos do gradiente e o aprendizado é feito por épocas. O ajuste dos pesos é realizado depois da apresentação completa 
de todo o conjunto padrão de treinamento à RNA.

\title{
Agradecimentos
}

Os autores agradecem o apoio financeiro do CNPq, Conselho Nacional de Desenvolvimento Científico e Tecnológico e da FAPERJ, Fundação Carlos Chagas Filho de Amparo à Pesquisa do Estado do Rio de Janeiro. Oliveira também agradece ao IF-RN, Instituto Federal do Estado do Rio Grande do Norte, à Secretaria de Estado de Tributação do Estado do Rio Grande do Norte e ao Governo do Estado do Rio Grande do Norte, pelo seu afastamento remunerado para cursar o Mestrado em Modelagem Computacional no Instituto Politécnico da UERJ.

\begin{abstract}
This work is based on the concept of Neural Networks Committee Machine and has the objective to solve the inverse radiative transfer problem in onedimensional, homogeneous, absorbing and isotropic scattering media. The Neural Networks Committee Machine combines the knowledge acquired by a number of specialists which are represented, individually, by each one of the Artificial Neural Networks (ANN) that composes the committee. The objective is to reach a final result hypothetically better than the ones obtained by any of the specialists separately. The use of this technique may reduce the computational time waste that occurs when several ANNs are trained, separately, being selected only the network that presents the best generalization and discarding the others. In the present work estimates for radiative transfer parameters related to the medium optical thickness, single scattering albedo and reflectivities are obtained using the technique of Neural Networks Committee Machine. Finally the results obtained are compared with those found using Multi-Layer Perceptron Networks (MLPs) individually, which are denominated specialist networks in the present work, showing that the technique used brings a performance improvement with a relatively low computational cost.
\end{abstract}

\section{Referências}

[1] W.J. Blackwell, A neural-network technique for retrieval of atmospheric temperature and moisture profiles from high spectral resolution sounding data, IEEE Trans Geosci Remote Sens, 43 (2005), 2535-2546.

[2] H.F. de Campos Velho, M.R. Rematoso, M.T. Vilhena, Inverse problems for estimating bottom boundary conditions of natural waters, Int $J$ Numer Meth Engng, 54(9)(2002), 1357-1368.

[3] H.F. de Campos Velho, J.D.S. Silva, E.H. Shiguemori, Hardware implementation for the atmospheric temperature retrieval from satellite data, Inverse Problems, Design and Optimization Symposium (IPDO) 1,(2007), 349-353.

[4] E.S. Chalhoub, H.F. de Campos Velho, Multispectral reconstruction of bioluminescence term in natural waters, Appl Numer Math, 47 (2003), 365-376.

[5] S. Chandrasekhar, "Radiative Transfer", Dover Publications Inc., New York, 1960.

[6] S. Haykin , "Neural Networks: A Comprehensive Foundation", Prentice Hall, 1994. 
[7] R.C. Oliveira, N.I. Alvarez Acevedo, A.J. Silva Neto, L. Biondi Neto, Aplicação da técnica de máquina de comitê de redes neurais artificiais para a solução de problemas inversos em transferência radiativa, XI Encontro de Modelagem Computacional, Volta Redonda, R.J., (2008).

[8] M.N. Özisik, "Radiative Transfer and Interactions with Conduction and Convection", John Wiley \& Sons, USA, (1973).

[9] M. Riedmiller, H. Braun, A direct adaptative method for faster backpropagation learning: the RPROP algorithm Proc. IEEE International Conference on Neural Networks, (1993), 234-241.

[10] A.J. Silva Neto, F.J.C.P. Soeiro, Inverse Problem of Space Dependent Albedo Estimation with Artificial Neural Networks and Hybrid Methods, Proc. 18th International Congress of Mechanical Engineering, COBEM, ABCM, Ouro Preto, Brazil, (2005).

[11] A.J. Silva Neto, F.J.C.P. Soeiro, H.F. Campos Velho, P. Oliva Soares, Using Neural Networks to Obtain Initial Estimates for the Solution of Inverse Heat Transfer Problems, Inverse Problems, Design and Optimization Symposium, Rio de Janeiro, Brazil,(2004).

[12] F.J.C.P. Soeiro, A.J. Silva Neto, Solution of Inverse Radiative Transfer Problems in Two-Layer Media with Artificial Neural Networks, $14^{\text {th }}$ Inverse Problems in Engineering Seminar, Ames, USA, (2006).

[13] F.J.C.P. Soeiro, P. Oliva Soares, A.J. Silva Neto, Solution of Inverse Radiative Transfer Problems with Artificial Neural Networks and Hybrid Methods, Proc. $13^{\text {th }}$ Inverse Problems in Engineering Seminar, Cincinnatti, USA, (2004), 163169 . 